\title{
IRREDUCIBLE INGUINAL HERNIA IN A FEMALE WITH INTRA-VAGINAL FOREIGN BODY: A CASE REPORT
}

Shanta B. Patil ${ }^{1}$, Avinash R. Odugudai ${ }^{2}$, Nagaraj Mallada ${ }^{3}$.

1. Associate Professor, Department Of General Surgery, Mahadevappa Ramdure Medical College, Gulbarga.

2. Resident, Department Of General Surgery, Mahadevappa Ramdure Medical College, Gulbarga.

3. Resident, Department Of General Surgery, Mahadevappa Ramdure Medical College, Gulbarga.

\section{CORRESPONDING AUTHOR:}

Dr .Shanta B. Patil, \#10-2/34 Sangameshwar Colony, SB College, Gulbarga-3, Karnataka.

E-mail: shantapatil44@gmail.com

\begin{abstract}
Inguinal hernia is the most common hernia seen in females. Only independent risk factors for inguinal hernia in females are positive family history and obstipation. Here we present a case of 60yr old female with irreducible inguinal hernia. On examination an intra-vaginal foreign body was found which was causing patient to strain during micturition leading to chronic increase in intra-abdominal pressure. A herniorrhaphy was done and foreign body was removed intraoperatively.
\end{abstract}

INTRODUCTION: Inguinal hernia in females is relatively uncommon as compared to males but inguinal hernia is the most common hernia in females. The incidence of inguinal hernia in females is $<5 \%$. Inguinal hernias are nine times more common in men than in women.[1] The incidence of indirect hernia relates to the congenital weakness at the internal abdominal ring. The sac is formed by the unobliterated portion of the prenatal peritoneal invagination of the canal of nuck that runs along and partly covers the round ligament. Virtually nothing is known about risk factors for inguinal hernia in females. High sports activity is protective in inguinal hernia. Smoking, appendicectomy, abdominal operations and multiple deliveries are not associated with inguinal hernia in females.[2] Inguinal hernia has been reported to be more prevalent in people with low socio-economic status. ${ }^{[3]}$ Inguinal hernias have an approximate incidence of incarceration of $10 \%$ and a portion of these may become strangulated. ${ }^{[4]}$ In a study, bowel resection rate in patients with obstructed or strangulated hernias was $15.9 \%$. The need for bowel resection closely correlated with the time interval between the onset of acute symptoms and subsequent operation. ${ }^{[5]}$ Immediate operation should be done in all patients who are ill with incarcerated or obstructed hernia, without attempting reduction.

CASE REPORT: A 60 year old female presented to our hospital with history of swelling in the right groin for the past 15 years which progressively increased in size. She also gave history of straining on passing urine and constipation. Patient was a known case of chronic obstructive pulmonary disease with 2-3 episodes of breathlessness per year. On examination right inguinal swelling extending from inguinal to vulval region and measuring $12 \times 10 \mathrm{cms}$ was found. There was no visible peristalsis and swelling was irreducible. Bowel sounds were heard over the swelling. Erect X-ray of 
the abdomen revealed a foreign body in the bony pelvis (figure 1). USG abdomen and pelvis showed foreign body in vaginal vault. All other investigations were within normal limits. Patient was taken for surgery (figure 2) and herniorrhaphy was done followed by extraction of the foreign body. Post -operative period was uneventful.

DISCUSSION: All inguinal hernias in females occur as indirect protrusions. Although no risk factors for inguinal hernias in females are known, independent risk factors are positive family history and obstipation. Protective effects of sports activity in females can be explained by optimising the resistance of the abdominal musculature protecting the relatively small inguinal weak point. [6] Because of the stress of child-bearing, the transversalis fascia is stronger in the floor of the inguinal canal and hence has protective effect, so direct hernia in females is unusual. [7]

It is commonly thought that repeated increases in intra-abdominal pressure contribute to hernia formation; hence, inguinal hernias are commonly associated with pregnancy, chronic obstructive pulmonary disease, abdominal ascites, patients who undergo peritoneal dialysis, labourers who repeatedly flex the abdominal wall musculature, and individuals who strain from constipation. It is also thought that collagen formation and structure deteriorates with age, and thus hernia formation is more common in the older individual. [8] Bladder neck obstruction causes retention of urine causing frequent urge to void urine and straining during micturition. Conditions leading to bladder neck obstruction in females are urethral stricture, pregnancy (retroverted gravid uterus), fibroids, ovarian cyst, carcinoma of the cervix uteri and rectum and any pelvic growth.

In our case the intra-uterine foreign body which was introduced to prevent uterine prolapse as a local practice caused partial obstruction at the bladder neck causing the patient to strain while micturition. Other factors like history of chronic cough, constipation and aging also contributed towards development of inguinal hernia.

\section{REFERENCES:}

1. McIntosh A, Hutchinson A, Roberts A, et al. Evidence-based management of groin hernia in primary careâ€”a systematic review. Fam Pract 2000;17:442 [PubMed: 11021907]

2. Liem MS. Graaf VY, Zwart RC el al. Risk factors for inguinal hernia in women: a case control study. Am J Epidemiology 1997; 146(9):72l-7.

3. Osifo OD, Amusan TI. Outcome of giant inguinoscrotal hernia repair with local lidocaine anaesthesia. Saudi Med J. 2010;31(1):53-58

4. The Washington manual of surgery, fifth edition, chapter $26 ; 442$

5. Mabula JB, Chalya PL. Surgical management of inguinal hernias at bugando medical centre in northwestern Tanzania: our experiences in a resource-limited setting. BMC Res Notes. 2012 Oct 25; 5:585. doi: 10.1186/1756-0500-5-585.

6. Kaplan SA, Synder WH, Little S. Inguinal hernia in females and testicular feminization syndrome. Am J Dis children 1969;117:43-51

7. Gnidec AA, Marshall DG. Incarcerated direct inguinal hernia containing uterus, both ovaries, and fallopian tubes. JPediatr Surg. 1986 Nov; 21(11):986.

8. Maingot's abdominal operations, $11^{\text {th }}$ edition; Chapter 5 . Hernias 


\section{CASE REPORT}

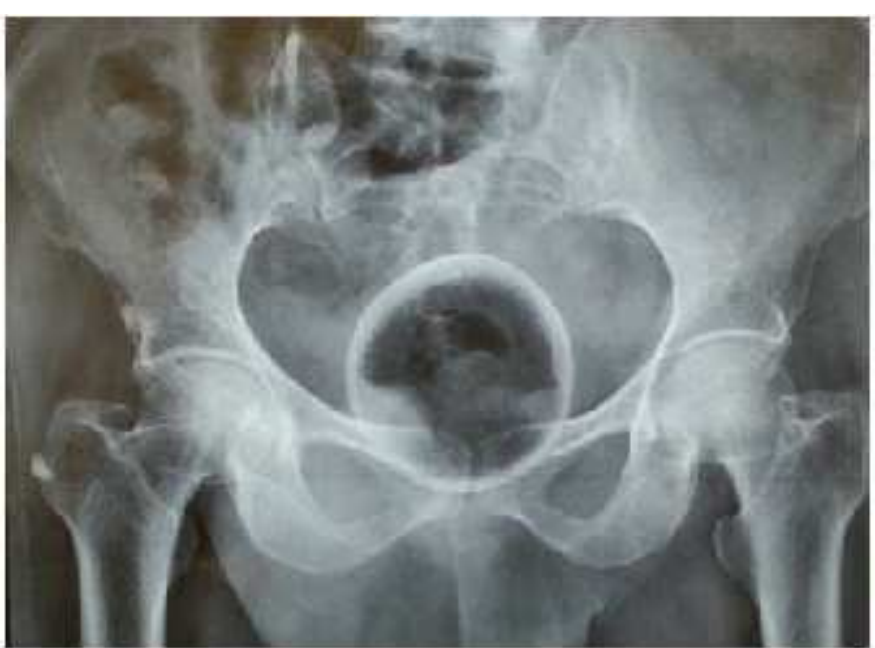

Figure 1: X-ray pelvis showing foreign body.

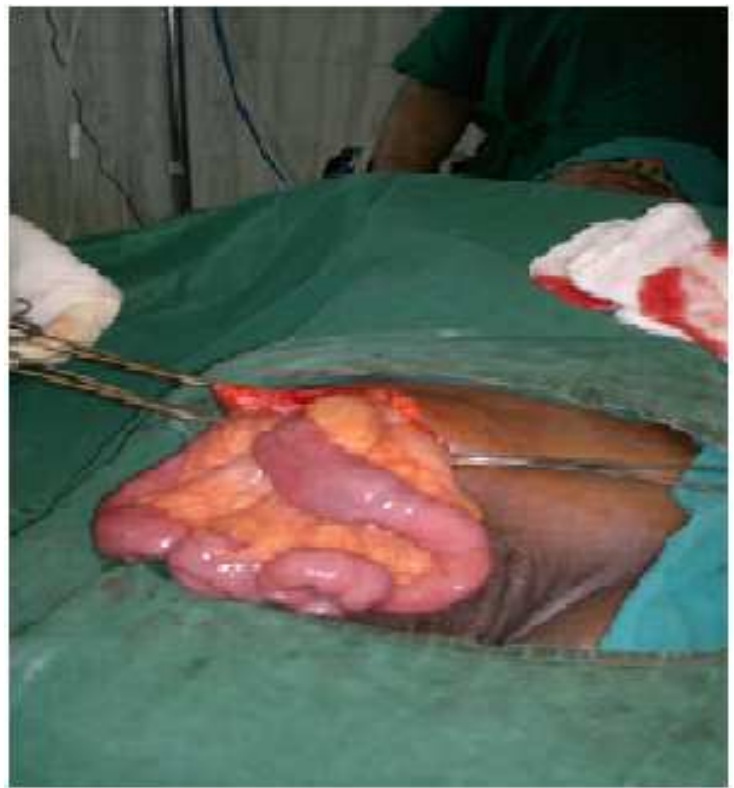

Figure 2: Intra-operative image showing the contents of the sac. 